\title{
The Risk of Acute Myocardial Infarction in Patients With Gastroesophageal Reflux Disease
}

\author{
Mohamed Eisa, ${ }^{1}$ Annumeet Sandhu, ${ }^{1}$ Ravi Prakash, ${ }^{1}$ Stephen J Ganocy, ${ }^{2}$ and Ronnie Fass ${ }^{1 *}$ \\ ${ }^{\prime}$ Department of Internal Medicine, The Esophageal and Swallowing Center, Division of Gastroenterology and Hepatology, MetroHealth \\ Medical Center, Case Western Reserve University, Cleveland, OH, USA; and ${ }^{2}$ Center for Health Care Research and Policy, Case Western Reserve \\ University, Cleveland, OH, USA
}

\section{Background/Aims}

A number of inflammatory mediators have been documented to be elevated in gastroesophageal reflux disease (GERD). Similar inflammatory mediators are involved in coronary artery disease. Thus, the aim of the study is to determine if GERD is a risk factor for developing acute myocardial infarction (AMI).

\section{Methods}

We used Explorys, a private cloud-based data store to which a number of health care systems feed information. We identified a cohort of GERD patients who have undergone an esophagogastroduodenoscopy compared to those without GERD. Incidence of AMI was studied after statistically controlling for known AMl risk factors.

\section{Results}

Total of 200400 patients were included in the GERD group and 386800 patients in non-GERD group. The primary event of AMI occurred in 17200 patients in the GERD group (8.6\%) vs 24300 in non-GERD group (6.3\%). Using logistic regression analysis and controlling for 6 major risk factors which included male gender (OR, 1.09; 95\% Cl, 1.07-1.11; $P<0.001)$, hypertension (OR, 6.53; $95 \% \mathrm{Cl}, 6.21-6.88 ; P<0.001)$, hyperlipidemia (OR, 3.08; 95\% Cl, 2.96-3.20; $P<0.001)$, diabetes mellitus (OR, 1.72; $95 \% \mathrm{Cl}, 1.69$ 1.76; $P<0.001)$, obesity $(\mathrm{OR}, 1.02 ; 95 \% \mathrm{Cl}, 1.00-1.04 ; P=0.044)$, and smoking $(\mathrm{OR}, 1.38 ; 95 \% \mathrm{Cl}, 1.35-1.41 ; P<0.001)$. The odds of developing AMI in the GERD population was $1.11(95 \% \mathrm{Cl}, 1.08-1.13 ; P<0.001)$. GERD had higher odds of developing AMI than male gender or obesity in our study.

\section{Conclusions}

This study demonstrated that GERD is a risk factor for AMI, higher than male gender and obesity. However, the increased risk may be clinically insignificant.

(J Neurogastroenterol Motil 2020;26:471-476)

\section{Key Words}

Cloud computing; Coronary artery disease; Gastroesophageal reflux disease; Inflammation mediators; Myocardial infarction

\section{Received: October 7, 2019 Revised: June 1, 2020 Accepted: June 9, 2020}

(a) This is an Open Access article distributed under the terms of the Creative Commons Attribution Non-Commercial License (http://creativecommons. org/licenses/by-nc/4.0) which permits unrestricted non-commercial use, distribution, and reproduction in any medium, provided the original work is properly cited.

*Correspondence: Ronnie Fass, MD Division of Gastroenterology and Hepatology, Esophageal and Swallowing Center, Case Western Reserve University, MetroHealth Medical Center, 2500 MetroHealth Drive, Cleveland, OH 44109, USA Tel: +1-216-778-3145, Fax: +1-216-778-2074,E-mail: ronnie.fass@gmail.com 


\section{Introduction}

Inflammation has long been recognized as a key factor in the pathogenesis of cardiovascular disease and formation of atherosclerosis. In the light of this fact, our study aims to evaluate if gastroesophageal reflux disease (GERD) which is a chronic inflammatory state (see below), as a risk factor for acute myocardial infarction (AMI). Coronary artery disease (CAD) is one of the most common cardiovascular illnesses in the United States (US) which results in significant morbidity and mortality. ${ }^{1}$ In 2009 alone over 1.2 million hospital discharges were associated with the diagnosis of acute coronary syndrome. ${ }^{2}$ This has a significant economic impact on the health care system with economic burden of close to 50 billion US dollars in direct health care costs just in 2015. ${ }^{3}$ There are multiple well established risk factors for the development of AMI. ${ }^{4} \mathrm{How}-$ ever, advances in research over the last 2 decades have established a more fundamental role for inflammation in mediating almost all stages of atherogenesis. ${ }^{5}$ These findings have enabled to identify certain chronic inflammatory processes as risk factors for coronary events. $^{6,7}$

GERD, a very common disorder, is primarily the disorder of the esophagogastric junction, directly related to esophageal acid exposure. ${ }^{8-11}$ However, recent advances have broadened our understanding of the role of immunopathogenesis in the disease and currently GERD is considered a chronic inflammatory disorder. When the esophageal epithelium is exposed to bile acid, trypsin, and pepsin in the presence of low $\mathrm{PH}$, proteinase-activated receptor-2 is activated. These receptors are located in all layers of the stratified squamous epithelium. ${ }^{12,13}$ There is marked inflammatory response following stimulation of these receptors. ${ }^{14}$ One of the more well studied cytokines in relation to GERD is IL-8. ${ }^{15,16}$ This cytokine, which is a powerful chemo-attracting compound of leukocytes and non-immune cells, is significantly increased in GERD patients. ${ }^{17,18}$ Similarly, there are other cytokines such as IL-1 $\beta$, IL-6, platelets activating factor, and reactive oxygen species that have been shown to be high in patients with GERD. ${ }^{19-21}$ These cytokines may also have a role in promoting esophageal fibrosis and even carcinogenesis. ${ }^{22,23}$ However, thus far there are no studies that evaluated the systemic impact of elevated inflammatory mediators in GERD.

It has been established that immune cells dominate formation of atherosclerotic lesions in the coronary arteries and elsewhere in the cardiovascular system. ${ }^{24,25}$ Their effector molecules (mainly IL-1, tumor necrosis factor- $\alpha$, and IL-6 inflammatory cytokines) can not only accelerate the progression of these lesions but also pre- cipitate acute coronary syndrome with plaque rupture. Thus, the same inflammatory cytokines that are elevated in GERD, are those involved in thrombus formation.

As a result, the aim of the current study is to compare the incidence of new AMIs in patients with GERD versus patients without GERD while controlling for the major ischemic heart disease risk factors.

\section{Materials and Methods}

In order to accomplish this, we performed a large national database study using a platform named "Explorys." Because most of the co-morbidities which increase the risk of AMI are also common in GERD patients, it was important to access a very large population database.

\section{Patients}

We identified a cohort of patients with a "Diagnosis: GERD," who have undergone an esophagogastroduodenoscopy (EGD). Explorys uses Current Procedural Terminology (CPT) codes from the American Medical Association data files. We ensured that GERD patients have undergone EGD within 1 week of the diagnosis. The control group was defined as those who never had a diagnosis of GERD. Then we included a cohort of patients between 18 to 85 years in either group. All patients had to be active in the electronic health system during the time of data collection. When studying the GERD group, we ensured the diagnosis of GERD was made prior to the primary outcome or the diagnosis of AMI. Patients were excluded if they had prior history of coronary event, whether AMI or stable coronary artery disease. Patients were also excluded if they had a diagnosis of peripheral artery disease. The study was approved by the Institutional Review Board (IRB No. 18-00532). We used the CPT code "acute myocardial infarction" as our primary outcome after reviewing all the definitions related to coronary artery disease (CAD), and we excluded the presence of CAD or AMI prior to the diagnosis of GERD to ensure the primary outcome occurred after the diagnosis of GERD. Patients with myocardial infarction were identified as those with the presence of Systematized Nomenclature of Medicine-Clinical Terms (SNOMED)-CT diagnosis of "acute myocardial infarction." To increase the internal validity of our results, we re-collected the data and repeated the analysis. That was especially important for the diagnosis of AMI since Explorys does not provide troponin levels or electrocardiographic (EKG) data. 


\section{Study Design}

This was a large nationwide database cohort study involving 2 groups. The study group was the GERD patients which were compared to those who did not have the diagnosis of GERD using the database platform "Explorys" (infra vide).

The primary outcome of the study was the incidence of new AMI in either group. By not including patients with prior coronary events we were ensuring that GERD preceded patients' coronary event. However, there are a number of confounding factors which could affect the association between GERD and AMI. Based on recent publication we identified the 6 most important risk factors which included; hypertension, hyperlipidemia, obesity, diabetes mellitus, male gender, and tobacco use. Obesity was defined as patients with body mass index that is greater than $30 \mathrm{~kg} / \mathrm{m}^{2}$. Subjects with hypertension, hyperlipidemia, diabetes mellitus, or current smoker were included based on available documentation in their electronic chart.

\section{The Explorys Database}

Explorys (www.explorys.com) is a private cloud-based data store established in 2009. Healthcare data span across a disparate ecosystem of employed and affiliated providers, payers and plans, care setting and electronic medical systems. Explorys is unique in a way that it ties all of these variables together.

De-identified data was obtained using the explore application of the Explorys platform. The data was obtained from a collection of health information systems which include billing inquires, electronic medical records, and others. A web application allows institutions to search and analyze the standardized, normalized, and de-identified data.

All data were de-identified to meet The Health Insurance Probability and Accountability Act and The Health Information Technology for Economic and Clinical Health Act standards. Business affiliation agreements were in place between all participating healthcare systems and Explorys Inc. regarding contribution of electronic medical records data and the use of de-identified data. Unified Medical Language Systems ontologies were used to map EHR data to facilitate searching and medical clinical terms [SNOMED-CT]) hierarchy. ${ }^{26}$ Prescription medication orders were mapped to RxNorm. ${ }^{27}$ Laboratory test observation were mapped to Logical Observation Identifiers Names and Code, established by the Regenstrief institute. ${ }^{28}$

\section{Explorys Data Retrieval}

There was aggregate level data available on Explorys. Since the system derives de-identified data from multiple health care systems across the country, individual level data are not available or obtainable. Only dichotomous variables were considered for analysis. All efforts were made to ensure that the data pool remained unchanged. Cohorts were defined based on a total of 8 variables which therein amounted to a total of 256 cohorts. These were individually defined and then entered to a total of 256 cohorts. They were individually defined and then entered into the Explorys search engine. Following this, queries were run to pull total counts and incidence of acute myocardial infarction for each cohort.

\section{Statistical Methods}

Data for the statistical analysis consisted of the 8 dichotomous categorical variables described above. Number and percentages were presented to describe the variables. Logistic regression analysis was used to assess the influence of the explanatory variables on the outcome variable AMI. Bonferroni correction was used to compensate for multiple testing. Due to the nuance of reporting counts in the Explorys system, which reports counts only to the nearest 10 , counts equal to zero were converted to the value 1 in order to maintain stability in the solution of the logistic regression equations. Statistical analysis was conducted using SAS software version 9.4. Copyright SAS institute Inc. SAS and all other SAS Institute Inc. product or service names are registered trademarks or trademarks of SAS Institute Inc, Cary, NC, USA.

\section{Results}

There were total of 200400 patients in the GERD group and 386800 patients in the non-GERD arm. Due to our inclusion and exclusion criteria, the number of GERD patients included in our study was less than $1 \%$ of the total Explorys population. The primary outcome which was the incidence of new AMI in the GERD group was 17200 cases resulting in an event rate of $8.6 \%$. In the non-GERD arm there were 24300 cases of AMI with an event rate of $6.3 \%$. The crude unadjusted odds ratio was 1.40 (95\% CI, 1.37-1.43)

Table 1 shows the demographics of patients with GERD and non-GERD. The total of patients diagnosed with GERD between the age range of 18 and 85 years were 200400 . There were 52.1\% men in the GERD group compared to $51.8 \%$ in the non-GERD group arm which are nearly comparable. There were $36.6 \%$ smok- 
Table 1. Demographics of Patients With Gastroesophageal Reflux Disease and Non-gastroesophageal Reflux Disease

\begin{tabular}{lcc}
\hline \multicolumn{1}{c}{ Variable } & GERD & Non-GERD \\
\hline Sex & & \\
$\quad$ Male & $52.1 \%$ & $51.8 \%$ \\
Female & $47.9 \%$ & $48.2 \%$ \\
Smoker & & \\
Yes & $36.6 \%$ & $32.1 \%$ \\
No & $63.4 \%$ & $67.9 \%$ \\
Mean age (yr) & $65-69$ & $75-79$ \\
Alcohol consumption & $42.0 \%$ & $47.0 \%$ \\
Race & & \\
Caucasian & $62.0 \%$ & $59.0 \%$ \\
$\quad$ Non-Caucasian & $38.0 \%$ & $41.0 \%$ \\
\hline
\end{tabular}

GERD, gastroesophageal reflux disease.

Table 2. Comparison of the Prevalence of Major Risk Factors for Coronary Artery Disease Between Gastroesophageal Reflux Disease and Non-gastroesophageal Reflux Disease Patients

\begin{tabular}{lcc}
\hline \multicolumn{1}{c}{ Characteristic } & $\begin{array}{c}\text { GERD } \\
(\mathrm{n}=200400)\end{array}$ & $\begin{array}{c}\text { Non-GERD } \\
(\mathrm{n}=386800)\end{array}$ \\
\hline AMI & $17200(8.6)$ & $24300(6.3)$ \\
Male & $104400(52.1)$ & $200310(51.8)$ \\
Hypertension & $156660(78.2)$ & $254060(65.7)$ \\
Hyperlipidemia & $157100(78.4)$ & $248370(64.2)$ \\
Diabetes & $74480(37.2)$ & $115410(29.8)$ \\
Obesity & $89260(44.5)$ & $132470(34.3)$ \\
Smoking & $73290(36.6)$ & $124020(32.1)$ \\
\hline
\end{tabular}

GERD, gastroesophageal reflux disease; AMI, acute myocardial infarction.

Data are presented as number (\%).

ers in the GERD group vs $32.1 \%$ in the non-GERD group.

Table 2 presents the prevalence of the 6 major risk factors for ischemic heart disease for the 2 patient groups (GERD vs nonGERD). Diabetes Mellitus (37.2\% vs 29.8\%), hypertension (78.2\% vs $65.7 \%)$, hyperlipidemia (78.4\% vs $64.2 \%)$, obesity (44.5\% vs $34.3 \%$ ), and smoking ( $36.6 \%$ vs $32.1 \%$ ), respectively.

Logistic regression was performed to assess the strength of the association between GERD and AMI (Table 3). After controlling for all 6 major risk factors which included male gender (OR, 1.09; 95\% CI, 1.07-1.11; $P<0.001)$, hypertension (OR, 6.53; 95\% CI, 6.21-6.88; $P<0.001$ ), hyperlipidemia (OR, 3.08; 95\% CI, 2.963.20; $P<0.001$ ), diabetes mellitus (OR, 1.72; 95\% CI, 1.69-1.76; $P<0.001$ ), obesity (OR, $1.02 ; 95 \% \mathrm{CI}, 1.00-1.04 ; P=0.044)$, and smoking (OR, 1.38; 95\% CI, 1.35-1.41; $P<0.001)$, the odds
Table 3. Logistic Regression Analysis of Acute Myocardial Infarction Status in the Patient Population Adjusted for Gastroesophageal Reflux Disease and Major Risk Factors for Coronary Artery Disease

\begin{tabular}{|c|c|c|c|c|c|}
\hline Rank & Effect & $\begin{array}{c}\text { Odds } \\
\text { ratio }\end{array}$ & $\begin{array}{l}95 \% \\
\text { LCL }\end{array}$ & $\begin{array}{r}95 \% \\
\text { UCL }\end{array}$ & $P$-value \\
\hline 1 & Hypertension & 6.53 & 6.21 & 6.88 & $<0.001$ \\
\hline 2 & Hyperlipidemia & 3.08 & 2.96 & 3.20 & $<0.001$ \\
\hline 3 & Diabetes & 1.72 & 1.69 & 1.76 & $<0.001$ \\
\hline 4 & Smoking & 1.38 & 1.35 & 1.41 & $<0.001$ \\
\hline 5 & GERD & 1.11 & 1.08 & 1.13 & $<0.001$ \\
\hline 6 & Male & 1.09 & 1.07 & 1.11 & $<0.001$ \\
\hline 7 & Obesity & 1.02 & 1.00 & 1.04 & 0.044 \\
\hline
\end{tabular}

LCL, lower confidence limit; UCL, upper confidence limit; GERD, gastroesophageal reflux disease.

Effects ranked by decreasing order of risk.

of developing AMI in the GERD population was $1.11(95 \% \mathrm{CI}$, $1.08-1.13 ; P<0.001)$.

Proton pump inhibitors (PPIs) are the most commonly prescribed agents for GERD as they are the most potent acid suppressing agents. In our study, $44.6 \%$ of the patients with GERD were prescribed PPI as compared with $33.5 \%$ of the patients in the non-GERD group. The odds ratio of AMI amongst GERD patients, when adjusted for PPI use was 1.11 compared to 1.10 for those who did not use PPI. Thus, PPI use did not remove the risk GERD posed for AMI. We concluded that the PPI prescribed for the non-GERD group was probably for non-GERD indications such as peptic ulcer disease, and patients on long term NSAIDs and steroids.

\section{Discussion}

In this large nationwide study, we examined GERD as a risk factor for AMI and coronary artery disease. The biological plausibility of our theory was based on the fact that GERD and CAD (eg, AMI) share the same immunopathogenesis and inflammatory cytokines. Therefore, we hypothesized that patients with GERD may have a higher incidence of CAD and thus AMI. While the odds ratio was 1.11, it was higher than some of the known AMI risk factors.

Our study showed that patients with GERD have a higher risk of developing AMI in comparison with the known major risk factors (hypertension, hyperlipidemia, diabetes, smoking, male gender, and obesity) for $\mathrm{CAD}$, after those risk factors were controlled statistically. Hypertension was by far the greatest risk factor for AMI in our cohort followed by hyperlipidemia, diabetes, and smoking. 
However, our study showed that, GERD as a risk factor for AMI was ranked above male gender and obesity.

The awareness for the association between GERD and CAD has been increasing recently. A similar, nationwide populationbased study in Taiwan demonstrated that GERD patients had higher incidence of AMI than those without GERD, and that GERD was independently associated with increased risk for subsequent myocardial infarction. ${ }^{29}$ In contrast, Johansson et $\mathrm{al}^{30}$ demonstrated that GERD was not an independent predictor of AMI, however an increased risk was observed in the immediate days after the initial diagnosis of GERD, but no excess risk was observed once this period of time was discounted. A small study using simultaneous 24-hour ambulatory esophageal $\mathrm{pH}$ monitoring, 7-lead EKG monitoring and documentation of symptoms in patients taking their usual anti-anginal medication, demonstrated that GERD occurs frequently in patients with CAD. ${ }^{31}$ Our hypothesis was based on the fact that GERD and CAD share the same immunopathogenesis and inflammatory cytokines, however Chauhan et $\mathrm{al}^{32}$ demonstrated that esophageal acid stimulation can produce angina and significantly reduce coronary blood flow in humans in a laboratory setting. A study by Dobrzycki et $\mathrm{al}^{33}$ which used simultaneous 24-hour continuous EKG and $\mathrm{pH}$ monitoring in an angiography proven CAD patients demonstrated that GERD patients had larger total ischemic burden and higher number of ST segment depression. Furthermore, short term use of PPI restored normal esophageal $\mathrm{pH}$, and significantly reduced myocardial ischemia. $^{33}$ Although Dobrzycki et $\mathrm{al}^{33}$ studied the effect of GERD in known CAD patients, in our study we ensured that all patients developed AMI after the diagnosis of GERD was made. However, it is known that in atherosclerosis it may take several years before plaques rupture which then leads to AMI. This supports the hypothesis of the ongoing inflammatory process shared by GERD and AMI, and that GERD plays a role in activation of this process. In support of this, a study demonstrated that IL-6 receptor antagonist could provide a therapeutic approach to prevent $\mathrm{CAD}{ }^{34}$

Our study showed that GERD poses a risk for AMI more than obesity and male gender. In line of what we found, Zhu et $\mathrm{al}^{35}$ in their meta-analysis demonstrated a significant association between overweight and AMI (OR, 1.27; 95\% CI, 1.21-1.33; $P<0.001)$ with similar results in obese patients (OR, 1.22; 95\% CI, 1.07-1.40; $P=0.003)$. Furthermore, a retrospective study demonstrated that there was a strong inverse linear relationship between body mass index and earlier age of first non-ST segment elevation myocardial infarction (NSTEMI). ${ }^{36}$

Our study has certain limitations. The results are based on the Explorys system, which originates from 26 major Healthcare systems and 360 hospitals. This data is de-identified and it may not reveal certain regional trends or represent the diversity in the US health care system. For our analysis, we identified cohorts of GERD patients with and without AMI. We were unable to perform propensity-score matching because the Explorys platform only provides population-level data and not individual cases. Also, GERD diagnosis was based on the term "Diagnosis: gastroesophageal reflux disease" without specifying symptoms severity. Thus, we could not determine the relationship between severity of GERD and risk of developing AMI. Furthermore, Explorys is susceptible to limited documentation by physicians, which can lead to error in data collection and confound analysis.

In conclusion, in this large nationwide study, we demonstrated a higher incidence of first time AMI in patients with GERD compared to patients without GERD, which was statistically significant but may not be clinically meaningful. Therefore, GERD is an unrecognized risk factor for the development of coronary artery disease. Furthermore, we were able to demonstrate that GERD is a risk factor for AMI, higher than obesity and male gender. Moreover, PPI use did not remove the risk GERD poses for AMI. Lei et $\mathrm{al}^{29}$ commented that patients who were prescribed PPIs for more than 1 year showed only slight decrease in the rate of the new AMIs. However, the confidence interval crossed 0 (95\% CI, 0.31 1.04 ) and the $P$-value was not significant (0.066), suggesting that PPIs likely had no impact on the rate of new AMIs. ${ }^{29}$ Further prospective studies are needed to examine the effect and the severity of GERD on the development of coronary artery disease, and whether early control of GERD would change the risk for AMI.

\section{Financial support: None.}

\section{Conflicts of interest: None.}

Author contributions: Ronnie Fass: study design, data analysis, and drafting and finalizing manuscript; Mohamed Eisa and Annumeet Sandhu: data collection, data analysis, and drafting and finalizing manuscript; Ravi Prakash: study design and drafting manuscript; and Stephen Ganocy: data analysis, drafting and finalizing manuscript, and statistical analysis.

\section{References}

1. Kolansky DM. Acute coronary syndromes: morbidity, mortality, and pharmacoeconomic burden. Am J Manag Care 2009;15(2 suppl):S36S41. 
2. Roger VL, Go AS, Lloyd-Jones DM, et al. Executive summary: heart disease and stroke statistics--2012 update: a report from the American Heart Association. Circulation 2012;125:188-197.

3. Heidenreich PA, Trogdon JG, Khavjou OA, et al. Forecasting the future of cardiovascular disease in the United States: a policy statement from the American Heart Association. Circulation 2011;123:933-944.

4. Yusuf S, Hawken S, Ounpuu S, et al. Effect of potentially modifiable risk factors associated with myocardial infarction in 52 countries (the INTERHEART study): case-control study. Lancet 2004;364:937-952.

5. Ross R. Atherosclerosis--an inflammatory disease. N Engl J Med 1999;340:115-126.

6. Douglas KM, Pace AV, Treharne GJ, et al. Excess recurrent cardiac events in rheumatoid arthritis patients with acute coronary syndrome. Ann Rheum Dis 2006;65:348-353.

7. Kremers HM, McEvoy MT, Dann FJ, Gabriel SE. Heart disease in psoriasis. J Am Acad Dermatol 2007;57:347-354.

8. Soll AH, FassR. Gastroesophageal reflux disease: presentation and assessment of a common, challenging disorder. Clin Cornerstone 2003;5:214; discussion 14-17.

9. El-Serag HB. Epidemiology of non-erosive reflux disease. Digestion 2008;78(suppl 1):6-10.

10. Katz PO, Gerson LB, Vela MF. Guidelines for the diagnosis and management of gastroesophageal reflux disease. Am J Gastroenterol 2013;108:308-328.

11. El-Serag HB, Sweet S, Winchester CC, Dent J. Update on the epidemiology of gastro-oesophageal reflux disease: a systematic review. Gut 2014;63:871-880.

12. Inci KA, Edebo A, Olbe L, Casselbrant A. Expression of proteaseactivated-receptor 2 (PAR-2) in human esophageal mucosa. Scand J Gastroenterol 2009;44:664-671.

13. Kandulski A, Wex T, Mönkemüller K, et al. Proteinase-activated receptor-2 in the pathogenesis of gastroesophageal reflux disease. Am J Gastroenterol 2010;105:1934-1943.

14. Isomoto H. Wang A, Mizuta Y, et al. Elevated levels of chemokines in esophageal mucosa of patients with reflux esophagitis. Am J Gastroenterol 2003;98:551-556.

15. Yoshida N, Uchiyama K, Kuroda M, et al. Interleukin-8 expression in the esophageal mucosa of patients with gastroesophageal reflux disease. Scand J Gastroenterol 2004;39:816-822.

16. Yoshida N. [Cytokine expression in GERD.] Nihon Rinsho 2007;65:813-821. [Japanese]

17. HaradaA, Mukaida N, Matsushima K. Interleukin 8 as a novel target for intervention therapy in acute inflammatory diseases. Mol Med Today 1996;2:482-489

18. Ben-Baruch A, Grimm M, Bengali K, et al. The differential ability of IL-8 and neutrophil-activating peptide- 2 to induce attenuation of chemotaxis is mediated by their divergent capabilities to phosphorylate CXCR2 (IL-8 receptor B). J Immunol 1997;158:5927-5933.

19. Souza RF. Bringing GERD Management up to PAR-2. Am J Gastroenterol 2010;105:1944-1946.
20. Altomare A, Ma J, Guarino MP, et al. Platelet-activating factor and distinct chemokines are elevated in mucosal biopsies of erosive compared with non-erosive reflux disease patients and controls. Neurogastroenterol Motil 2012;24:943-e463.

21. Altomare A, Guarino MP, Cocca S, Emerenziani S, Cicala M. Gastroesophageal reflux disease: update on inflammation and symptom perception. World J Gastroenterol 2013;19:6523-6528.

22. Corley DA, Kerlikowske K, Verma R, Buffler P. Protective association of aspirin/NSAIDs and esophageal cancer: a systematic review and metaanalysis. Gastroenterology 2003;124:47-56.

23. Verbeke H, Geboes K, Van Damme J, Struyf S. The role of CXC chemokines in the transition of chronic inflammation to esophageal and gastric cancer. Biochim Biophys Acta 2012;1825:117-129.

24. Libby P, Ridker PM, Maseri A. Inflammation and atherosclerosis. Circulation 2002;105:1135-1143.

25. Deguchi JO, Aikawa M, Tung $\mathrm{CH}$, et al. Inflammation in atherosclerosis: visualizing matrix metalloproteinase action in macrophages in vivo. Circulation 2006;114:55-62.

26. Lee D, de Keizer N, Lau F, Cornet R. Literature review of SNOMED CT use. J Am Med Inform Assoc 2014;21:e11-e19.

27. Nelson SJ, Zeng K, Kilbourne J, Powell T, Moore R Normalized names for clinical drugs: RxNorm at 6 years. J Am Med Inform Assoc 2011;18:441-448.

28. McDonald CJ, Huff SM, Suico JG, et al. LOINC, a universal standard for identifying laboratory observations: a 5 -year update. Clin Chem 2003;49:624-633.

29. Lei WY, Wang JH, Wen SH, et al. Risk of acute myocardial infarction in patients with gastroesophageal reflux disease: a nationwide populationbased study. PLoS One 2017;12:e0173899.

30. Johansson S, Wallander MA, Ruigómez A, García Rodríguez LA. Is there any association between myocardial infarction, gastro-oesophageal reflux disease and acid-suppressing drugs? Aliment Pharmacol Ther 2003;18:973-978.

31. Mehta AJ, de Caestecker JS, Camm AJ, Northfield TC. Gastro-oesophageal reflux in patients with coronaryartery disease: how common is it and does it matter? Eur J Gastroenterol Hepatol 1996;8:973-978.

32. Chauhan A, Petch MC, Schofield PM. Cardio-oesophageal reflex in humans as a mechanism for 'linked angina'. Eur Heart J 1996;17:407413.

33. Dobrzycki S, Baniukiewicz A, Korecki J, et al. Does gastro-esophageal reflux provoke the myocardial ischemia in patients with CAD? Int J Cardiol 2005;104:67-72.

34. Swerdlow DI, Holmes MV, Kuchenbaecker KB, et al. The interleukin-6 receptor as a target for prevention of coronary heart disease: a mendelian randomisation analysis. Lancet 2012;379:1214-1224.

35. Zhu J, Su X, Li G, Chen J, Tang B, Yang Y. The incidence of acute myocardial infarction in relation to overweight and obesity: a meta-analysis. Arch Med Sci 2014;10:855-862.

36. Madala MC, Franklin BA, Chen AY, et al. Obesity and age of first nonST-segment elevation myocardial. J AM Coll Cardiol 2008;52:979-985. 